\title{
Application of Laplace-Adomian Decomposition Method for the Analytical Solution of Third-Order Dispersive Fractional Partial Differential Equations
}

\author{
Rasool Shah ${ }^{1,+(\mathbb{C}) \text {, Hassan Khan }}{ }^{1,+(\mathbb{C}, \text { Muhammad Arif }}{ }^{1,+}$, Poom Kumam ${ }^{2,3, *,+}$ \\ 1 Department of Mathematics, Abdul Wali khan University, Mardan 23200, Pakistan; \\ shahrasool26@gmail.com (R.S.); hassanmath@awkum.edu.pk (H.K.); marifmaths@awkum.edu.pk (M.A.) \\ 2 Center of Excellence in Theoretical and Computational Science (TaCS-CoE) \& KMUTTFixed Point Research \\ Laboratory, Room SCL 802 Fixed Point Laboratory, Science Laboratory Building, Departments of \\ Mathematics, Faculty of Science, King Mongkut's University of Technology Thonburi (KMUTT), \\ 126 Pracha-Uthit Road, Bang Mod, Thrung Khru, Bangkok 10140, Thailand \\ 3 Department of Medical Research, China Medical University Hospital, China Medical University, \\ Taichung 40402, Taiwan \\ * Correspondence: poom.kum@kmutt.ac.th \\ + These authors contributed equally to this work.
}

Received: 22 February 2019; Accepted: 24 March 2019; Published: 28 March 2019

check for updates

\begin{abstract}
In the present article, we related the analytical solution of the fractional-order dispersive partial differential equations, using the Laplace-Adomian decomposition method. The Caputo operator is used to define the derivative of fractional-order. Laplace-Adomian decomposition method solutions for both fractional and integer orders are obtained in series form, showing higher convergence of the proposed method. Illustrative examples are considered to confirm the validity of the present method. The fractional order solutions that are convergent to integer order solutions are also investigated.
\end{abstract}

Keywords: Laplace-Adomian decomposition method; third-order dispersive equations; Caputo operator; analytical solution

\section{Introduction}

Partial differential equations (PDEs) are used to model different physical phenomena in various areas of applied sciences such as fluid dynamics, mathematical biology, quantum mechanics, chemical kinetics and linear optics. In 1895, the Korteweg-de and Vries derived a non dimensionalized version of an equation, known as the KdV equation. This model is used for the study of dispersive wave phenomena in numerous fields of science and technology, like plasma physics and quantum mechanics. The exact solution of the $\mathrm{KdV}$ may not be available, therefore a lot of analytical techniques have been discussed for its approximate solution [1]. There are two important dispersive terms, namely third and fifty order in $\mathrm{KdV}$ equations. The $\mathrm{KdV}$ equation of order five has been used to describe plasma physics [2]. Numerical solutions of the fifth and third order dispersive KdV equations have been studied in [3].

The non-linear nature is responsible for the complete study of any physical system, which shows the importance of a non-linear term present in any model of the physical problems. In this connection, reductive perturbation theories have been studied in [4] for the non-linear KdV. A variational method has been suggested in [5] for the exact solution of $\mathrm{KdV}$ with higher-order nonlinearity. Numerical solutions for the KdV Burgers equation have been successfully derived in [6] by using a compact-type constrained interpolation profile method. Numerical results of KdV equations of order five are 
presented in [1] using homotopy perturbation transform method. The KdV equations of order three and five have been discussed in [3] by using two numerical methods. Entropies based on fractional calculus [7], integer and fractional dynamical systems can be solved by entropy analysis [8], nonlinear partial differential equations [9] in entropy and convexity, as well as ractional derivative advection-diffusion in two-dimensional semi-conductor systems and the dynamics of a national soccer league $[10,11]$. The exact solution to differential equations (DEs) of fractional order with mixed partial derivatives [12] are fractional linear differential equations with constant coefficients in Laplace transform [13]. Laplace homotopy analysis method (LHAM) can be used to solve FDEs [14] systems of non-linear FPDEs in a new analytical technique [15]. The solution to non-linear coupled space-time fractional modified KdV equations was obtained by Feng's first integral method [16]. Fractional PDEs of order three have been solved by different methods, such as fractional variational iteration method (FVIM) [17], classical Riccati equations method [18], fractional differential transform method(FDTM) and modified fractional differential transform method (MFDTM) [19], spline method (SM) [20] and homotopy analysis Sumudu transform method (HASTM) [21].

Among all these methods, the Laplace-Adomian decomposition method (LADM) is an efficient analytical method to solve non-linear FDES. LADM is the combination of two powerful techniques, the Adomian decomposition method and the Laplace transform. Further, LADM does not requireme the predefined size declaration like the Runge-Kutta method. Therefor this technique is considered, an ideal for those equations that represent nonlinear models. As compared to other analytical techniques, LADM have less parameters, therefore LADM is an efficient technique, requiring no discretization and linearization [22]. A comparison between the LADM and ADM for the analysis of FDEs is given in [23]. The Kundu-Eckhaus equation deals with quantum field theory, and the analytical solution of this nonlinear PDEs has been studied in [24] using LADM. Multi-step Laplace Adomian decomposition method have been described in [25] for non-linear FDEs. Analysis of smoke model have been studied successfully by using LADM [26].

In view of the above literature, we made a successful attempt to obtain the analytical results of dispersive FPDEs, using LADM. The results of the LADM are interesting and in better contact with exact solutions for the problems.

\section{Definitions and Preliminaries of Concepts}

In this unit, among a few definitions of fractional calculus, presented in the article due to Riemann Liouville, Grunewald Letnikov, Caputo, etc., first folk's simple descriptions and introductions are reconsidered, which we want to comprehend for our education.

Definition 1. $R-L$ fractional integral

$$
I_{x}^{\gamma} g(x)= \begin{cases}g(x) & \text { if } \gamma=0 \\ \frac{1}{\Gamma(\gamma)} \int_{0}^{x}(x-v)^{\gamma-1} g(v) d v & \text { if } \gamma>0,\end{cases}
$$

where $\Gamma$ denotes the gamma function define by,

$$
\Gamma(\omega)=\int_{0}^{\infty} e^{-x} x^{\omega-1} d x \quad \omega \in \mathbb{C},
$$

In this study, Caputo et al. [27] suggested a revise fractional derivative operator in order to overcome inconsistency measured in the Riemann Liouville derivative [27,28]. The above mathematical statement described a Caputo fractional derivative operator of initial and boundary condition for fractional as well as integer order derivative. 
Definition 2. The Caputo operator of order $\gamma$ for a fractional derivative is given by the following mathematical expression for $n \in \mathbb{N}, x>0, g \in \mathbb{C}_{t}, t \geq-1$.

$$
D^{\gamma} g(x)=\frac{\partial^{\gamma} g(x)}{\partial t^{\gamma}}=\left\{\begin{array}{l}
I^{n-\gamma}\left[\frac{\partial^{\gamma} g(x)}{\partial t^{\gamma}}\right], \quad \text { if } n-1<\gamma \leq n, n \in \mathbb{N} \\
\frac{\partial^{\gamma} g(x)}{\partial t^{\gamma}},
\end{array}\right.
$$

Hence, we require the subsequent properties given in the next Lemma.

Lemma 1. If $n-1<\gamma \leq n$ with $n \in \mathbb{N}$ and $g \in \mathbb{C}_{t}$ with $t \geq-1$, then

$$
\begin{aligned}
& I^{\gamma} I^{a} g(x)=I^{\gamma+a} g(x), \quad a, \gamma \geq 0 . \\
& I^{\gamma} x^{\lambda}=\frac{\Gamma(\lambda+1)}{\Gamma(\gamma+\lambda+1)} x^{\gamma+\lambda}, \quad \gamma>0, \lambda>-1, \quad x>0 . \\
& I^{\gamma} D^{\gamma} g(x)=g(x)-\sum_{k=0}^{n-1} g^{(k)}\left(0^{+}\right) \frac{x^{k}}{k !}, \quad \text { for } \quad x>0, n-1<\gamma \leq n .
\end{aligned}
$$

In current study, the Caputo operator is reasonable as other fractional derivative operators have certain disadvantages. Further information about fractional derivatives, are found in $[29,30]$.

Definition 3. The Laplace transform of $h(t), t>0$ is defined by [31]

$$
H(s)=\mathcal{L}[h(t)]=\int_{0}^{\infty} e^{-s t} h(t) d t .
$$

Definition 4. The Laplace transform in term of convolution is given by

$$
\mathcal{L}\left[h_{1} * h_{2}\right]=\mathcal{L}\left[h_{1}(t)\right] * \mathcal{L}\left[h_{2}(t)\right],
$$

here $h_{1} * h_{2}$ define the convolution between $h_{1}$ and $h_{2}$,

$$
\left(h_{1} * h_{2}\right) t=\int_{0}^{\tau} h_{1}(\tau) h_{2}(t-\tau) d t .
$$

The fractional derivative in term of a Laplace transform is

$$
\mathcal{L}\left(D_{t}^{\gamma} h(t)\right)=s^{\gamma} H(s)-\sum_{k=0}^{n-1} s^{\gamma-1-k} h^{(k)}(0), \quad n-1<\gamma<n,
$$

where $H(s)$ is the Laplace transform of $h(t)$.

Definition 5. The Mittag-Leffler function, $E_{\gamma}(p)$ for $\gamma>0$ is represented as,

$$
E_{\gamma}(p)=\sum_{n=0}^{\infty} \frac{p^{n}}{\Gamma(\gamma n+1)} \quad \gamma>0, \quad p \in \mathbb{C} .
$$

Theorem 1. Here, we will study the convergence analysis as same manner in [32] of the LADM applied to the fractional dispersive PDE of order three. Let us consider the Hilbert space $H$ which may define by $H=L^{2}((\alpha, \beta) X[0, T])$ the set of applications:

$$
u:(\alpha, \beta) X[0, T] \rightarrow \text { with } \int_{(\alpha, \beta) X[0, T]} u^{2}(x, s) d s d \theta \quad<+\infty .
$$


Now we consider the fractional dispersive PDE of order three in the above assumptions and let us denote

$$
L(u)=\frac{\partial^{\gamma} u}{\partial t^{\gamma}}
$$

then the fractional dispersive PDE becomes, in an operator form

$$
L(u)=-\varphi \frac{\partial v(x, t)}{\partial x}-w \frac{\partial^{3} v(x, t)}{\partial x^{3}} .
$$

The LADM is convergence, if the following two hypotheses are satisfied:

$$
(H 1)(L(u)-L(v), u-v) \geq k\|u-v\|^{2} ; k>0, \forall u, v \in H .
$$

$H(2)$ whatever may be $M>0$, there exist a constant $C(M)>0$ such that for $u, v \in H$ with $\|u\| \leq M$, $\|v\| \leq M$ we have $(L(u)-L(v), u-v) \leq C(M)\|u-v\|\|w\|$ for every $w \in H$.

\section{Idea of Fractional Laplace-Adomian Decomposition Method}

\subsection{LADM for Dispersive Equation of One-Dimensional}

In this section, LADM is implemented to solve fractional dispersive PDE of order three.

$$
\frac{\partial^{\gamma} v(x, t)}{\partial t^{\gamma}}+w \frac{\partial^{3} v(x, t)}{\partial x^{3}}=q(x, t), \quad w, t \geq 0, \quad m-1<\gamma<m,
$$

$q(x, t)$ is the source function.

Subject to initial and boundary conditions

$$
\begin{aligned}
& v(x, 0)=k(x), \\
& v(0, t)=l_{0}(t), \\
& v_{x}(0, t)=l_{1}(t), \\
& v_{x x}(0, t)=l_{2}(t) .
\end{aligned}
$$

With the help of Laplace transform, Equation (1) can be written as

$$
\begin{gathered}
\mathcal{L}\left[\frac{\partial^{\gamma} v(x, t)}{\partial t^{\gamma}}\right]+\mathcal{L}\left[w \frac{\partial^{3} v(x, t)}{\partial x^{3}}\right]=\mathcal{L}[q(x, t)], \\
\mathcal{L}[v(x, t)]=\frac{k(x)}{s}+\frac{1}{s^{\gamma}} \mathcal{L}[q(x, t)]-\frac{1}{s^{\gamma}} \mathcal{L}\left[w \frac{\partial^{3} v(x, t)}{\partial x^{3}}\right] .
\end{gathered}
$$

The LADM solution $v(x, t)$ is represented by the following infinite series

$$
v(x, t)=\sum_{j=0}^{\infty} v_{j}(x, t)
$$

and the nonlinear terms (if any) in problem are defined by the infinite series of Adomian polynomials,

$$
\begin{gathered}
N v(x, t)=\sum_{j=0}^{\infty} A_{j}, \\
A_{j}=\frac{1}{j !}\left[\frac{d^{j}}{d \lambda^{j}}\left[N \sum_{j=0}^{\infty}\left(\lambda^{j} v_{j}\right)\right]\right]_{\lambda=0}, \quad j=0,1,2 \ldots,
\end{gathered}
$$


and substitution of Equations (5) and (6) into Equation (4), we get

$$
\mathcal{L}\left[\sum_{j=0}^{\infty} v(x, t)\right]=\frac{k(x)}{s}+\frac{1}{s^{\gamma}} \mathcal{L}[q(x, t)]-\frac{1}{s^{\gamma}} \mathcal{L}\left[w \frac{\partial^{3} v_{j}(x, t)}{\partial x^{3}}\right] .
$$

Applying the linearity of the Laplace transform,

$$
\begin{gathered}
\mathcal{L}\left[v_{0}(x, t)\right]=\frac{v(x, 0)}{s}+\frac{1}{s^{\gamma}} \mathcal{L}[q(x, t)]=k(x, s), \\
\mathcal{L}\left[v_{1}(x, t)\right]=-\frac{1}{s^{\gamma}} \mathcal{L}\left[w \frac{\partial^{3} v_{0}(x, t)}{\partial x^{3}}\right] .
\end{gathered}
$$

Generally, we can write

$$
\mathcal{L}\left[v_{j+1}(x, t)\right]=-\frac{1}{s^{\gamma}} \mathcal{L}\left[\frac{\partial^{3} v_{j}(x, t)}{\partial x^{3}}\right], \quad j \geq 1 .
$$

Applying the inverse Laplace transform in Equation (9)

$$
\begin{gathered}
v_{0}(x, t)=k(x, t) \\
v_{j+1}(x, t)=-\mathcal{L}^{-1}\left[\frac{1}{s^{\gamma}} \mathcal{L}\left[\frac{\partial^{3} v_{j}(x, t)}{\partial x^{3}}\right]\right] .
\end{gathered}
$$

3.2. LADM for Dispersive Equation of Higher-Dimension

A dispersive PDE in higher dimension is represented as,

$$
\begin{gathered}
v_{t}^{\gamma}+c v_{x x x}+d v_{y y y}+e v_{z z z}=q(x, y, z, t) \\
t \geq 0, \quad c, d, e \geq 0, \quad m-1<\gamma<m
\end{gathered}
$$

where the source function is denoted by $q(x, y, z, t)$. The initial condition is

$$
v(x, y, z, 0)=k(x, y, z) .
$$

With the help of a Laplace transform, Equation (12) can be written as

$$
\mathcal{L}\left[v_{t}^{\gamma}\right]+\mathcal{L}\left[c v_{x x x}+d v_{y y y}+e v_{z z z}\right]=\mathcal{L}[q(x, y, z, t)],
$$

and using the differentiation property of the Laplace transform, we get

$$
\begin{aligned}
& \mathcal{L}\left[\sum_{j=0}^{\infty} v(x, y, z, t)\right]=\frac{k(x, y, z)}{s}+\frac{1}{s^{\gamma}} \mathcal{L}[q(x, y, z, t)] \\
& -\frac{1}{s^{\gamma}} \mathcal{L}\left[c v_{x x x}+d v_{y y y}+e v_{z z z}\right] .
\end{aligned}
$$

Applying the linearity of the Laplace transform,

$$
\begin{gathered}
\mathcal{L}\left[v_{0}(x, y, z, t)\right]=\frac{v(x, y, z, 0)}{s}+\frac{1}{s^{\gamma}} \mathcal{L}[q(x, y, z, t)]=k(x, y, z, s), \\
\mathcal{L}\left[v_{1}(x, y, z, t)\right]=-\frac{1}{s^{\gamma}} \mathcal{L}\left[c v_{0 x x x}+d v_{0 y y y}+e v_{0 z z z}\right] .
\end{gathered}
$$


Generally, we can write

$$
\mathcal{L}\left[v_{j+1}(x, y, z, t)\right]=-\frac{1}{s^{\gamma}} \mathcal{L}\left[c v_{j x x x}+d v_{j y y y}+e v_{j z z z}\right], \quad j \geq 1 .
$$

Applying the inverse Laplace transform, in Equation (15)

$$
\begin{gathered}
v_{0}(x, y, z, t)=k(x, y, z, t) \\
v_{j+1}(x, y, z, t)=-\mathcal{L}^{-1}\left[\frac{1}{s^{\gamma}} \mathcal{L}\left[c v_{j x x x}+d v_{j y y y}+e v_{j z z z}\right]\right] .
\end{gathered}
$$

\section{Results}

Example 1. Consider the following fractional dispersive KdV in Equation [33]

$$
\frac{\partial^{\gamma} v}{\partial t^{\gamma}}+2 \frac{\partial v}{\partial x}+\frac{\partial^{3} v}{\partial x^{3}}=0, \quad t>0, \quad 0<\gamma \leq 1
$$

with the initial condition

$$
v(x, 0)=\sin x .
$$

Taking the Laplace transform of Equation (18),

$$
\begin{gathered}
\mathcal{L}\left[\frac{\partial^{\gamma} v}{\partial t^{\gamma}}\right]=-\mathcal{L}\left[2 \frac{\partial v}{\partial x}+\frac{\partial^{3} v}{\partial x^{3}}\right], \\
s^{\gamma} \mathcal{L}[v(x, t)]-s^{\gamma-1}[v(x, 0)]=-\mathcal{L}\left[2 \frac{\partial v}{\partial x}+\frac{\partial^{3} v}{\partial x^{3}}\right] .
\end{gathered}
$$

Applying the inverse Laplace transform

$$
\begin{gathered}
v(x, t)=\mathcal{L}^{-1}\left[\frac{v(x, 0)}{s}-\frac{1}{s^{\gamma}} \mathcal{L}\left[2 \frac{\partial v}{\partial x}+\frac{\partial^{3} v}{\partial x^{3}}\right]\right], \\
v(x, t)=\sin x-\mathcal{L}^{-1}\left[\frac{1}{s^{\gamma}} \mathcal{L}\left[2 \frac{\partial v}{\partial x}+\frac{\partial^{3} v}{\partial x^{3}}\right]\right] .
\end{gathered}
$$

Using the ADM procedure, we get

$$
\begin{gathered}
\sum_{j=0}^{\infty} v_{j}(x, t)=\sin x-\mathcal{L}^{-1}\left[\frac{1}{s^{\gamma}} \mathcal{L}\left[2 \sum_{j=0}^{\infty} \frac{\partial v_{j}}{\partial x}+\sum_{j=0}^{\infty} \frac{\partial^{3} v_{j}}{\partial x^{3}}\right]\right], \\
v_{0}(x, t)==\sin x \\
v_{j+1}(x, t)=-\mathcal{L}^{-1}\left[\frac{1}{s^{\gamma}} \mathcal{L}\left[2 \sum_{j=0}^{\infty} \frac{\partial v_{j}}{\partial x}+\sum_{j=0}^{\infty} \frac{\partial^{3} v_{j}}{\partial x^{3}}\right]\right]
\end{gathered}
$$


for $j=0,1,2, .$.

$$
\begin{aligned}
& v_{1}(x, t)=-\mathcal{L}^{-1}\left[\frac{1}{s^{\gamma}} \mathcal{L}\left[2 \frac{\partial v_{0}}{\partial x}+\frac{\partial^{3} v_{0}}{\partial x^{3}}\right]\right] \\
& v_{1}(x, t)=-\mathcal{L}^{-1}\left[\frac{\cos x}{s^{\gamma+1}}\right]=-\cos x \frac{t^{\gamma}}{\Gamma(\gamma+1)}, \\
& v_{2}(x, t)=-\mathcal{L}^{-1}\left[\frac{1}{s^{\gamma}} \mathcal{L}\left[2 \frac{\partial v_{1}}{\partial x}+\frac{\partial^{3} v_{1}}{\partial x^{3}}\right]\right], \\
& v_{2}(x, t)=-\mathcal{L}^{-1}\left[\frac{\sin x}{s^{2} \gamma+1}\right]=-\sin x \frac{t^{2 \gamma}}{\Gamma(2 \gamma+1)} .
\end{aligned}
$$

The subsequent terms are

$$
\begin{aligned}
& v_{3}(x, t)=-\mathcal{L}^{-1}\left[\frac{1}{s^{\gamma}} \mathcal{L}\left[2 \frac{\partial v_{2}}{\partial x}+\frac{\partial^{3} v_{2}}{\partial x^{3}}\right]\right], \\
& v_{3}(x, t)=-\mathcal{L}^{-1}\left[\frac{\cos x}{s^{3 \gamma+1}}\right]=\cos x \frac{t^{3 \gamma}}{\Gamma(3 \gamma+1)} .
\end{aligned}
$$

The LADM solution for Example 1 is

$$
\begin{gathered}
v(x, t)=v_{0}(x, t)+v_{1}(x, t)+v_{2}(x, t)+v_{3}(x, t)+\ldots, \\
v(x, t)=\sin x-\cos x \frac{t^{\gamma}}{\Gamma(\gamma+1)}-\sin x \frac{t^{2 \gamma}}{\Gamma(2 \gamma+1)}+\cos x \frac{t^{3 \gamma}}{\Gamma(3 \gamma+1)}+\ldots,
\end{gathered}
$$

and so on. The solution in a series form is given by

$$
\begin{aligned}
& v(x, t)=\sin x\left(1-\frac{t^{2 \gamma}}{\Gamma(2 \gamma+1)}+\frac{t^{4 \gamma}}{\Gamma(4 \gamma+1)}-\ldots\right) \\
& -\cos x\left(\frac{t^{\gamma}}{\Gamma(\gamma+1)}-\frac{t^{3 \gamma}}{\Gamma(3 \gamma+1)}+\frac{t^{5 \gamma}}{\Gamma(5 \gamma+1)}-\ldots\right)
\end{aligned}
$$

when $\gamma=1$, then the LADM solution is

$$
v(x, t)=\sin (x-t) .
$$

Figure 1 consists of four graphs; (a) the exact solution of $v(x, t)$ and (b) LADM solution $v(x, t)$ of Example 1 at $\gamma=1$. Figure $1 \mathrm{a}, \mathrm{b}$ indicate that the present method has strong agreement with the exact solution for the problem. In Figure 1c, d, two graphs (c) and (d) are given, that represent the analytical solution of Example 1 at fractional $\gamma=0.75$ and 0.50 , respectively. Figure 1c,d reflects that fractional-order approaches to integer order solution surfaces of fractional order are convergent to the integer order surface. It means that physically we can model any of the surfaces as desired by the physical phenomena occurring in nature.

Example 2. We next consider the following fractional dispersive KdV equation [33]

$$
\frac{\partial^{\gamma} v}{\partial t^{\gamma}}+\frac{\partial^{3} v}{\partial x^{3}}+\frac{\partial^{3} v}{\partial y^{3}}=0, \quad t>0, \quad 0<\gamma \leq 1,
$$

with initial condition

$$
v(x, y, 0)=\cos (x+y) .
$$


Taking Laplace transform of Equation (25),

$$
\begin{gathered}
\mathcal{L}\left[\frac{\partial^{\gamma} v}{\partial t^{\gamma}}\right]=-\mathcal{L}\left[\frac{\partial^{3} v}{\partial x^{3}}+\frac{\partial^{3} v}{\partial y^{3}}\right], \\
s^{\gamma} \mathcal{L}[v(x, y, t)]-s^{\gamma-1}[v(x, y, 0)]=-\mathcal{L}\left[\frac{\partial^{3} v}{\partial x^{3}}+\frac{\partial^{3} v}{\partial y^{3}}\right] .
\end{gathered}
$$

Applying the inverse Laplace transform

$$
\begin{aligned}
& v(x, y, t)=\mathcal{L}^{-1}\left[\frac{v(x, y, 0)}{s}-\frac{1}{s^{\gamma}} \mathcal{L}\left[\frac{\partial^{3} v}{\partial x^{3}}+\frac{\partial^{3} v}{\partial y^{3}}\right]\right], \\
& v(x, y, t)=\cos (x+y)-\mathcal{L}^{-1}\left[\frac{1}{s^{\gamma}} \mathcal{L}\left[\frac{\partial^{3} v}{\partial x^{3}}+\frac{\partial^{3} v}{\partial y^{3}}\right]\right] .
\end{aligned}
$$

Using ADM procedure, we get

$$
\begin{gathered}
\sum_{j=0}^{\infty} v_{j}(x, y, t)=\cos (x+y)-\mathcal{L}^{-1}\left[\frac{1}{s^{\gamma}} \mathcal{L}\left[\sum_{j=0}^{\infty} \frac{\partial^{3} v_{j}}{\partial x^{3}}+\sum_{j=0}^{\infty} \frac{\partial^{3} v_{j}}{\partial y^{3}}\right]\right], \\
v_{0}(x, y, t)=\cos (x+y) \\
v_{j+1}(x, y, t)=-\mathcal{L}^{-1}\left[\frac{1}{s^{\gamma}} \mathcal{L}\left[\sum_{j=0}^{\infty} \frac{\partial^{3} v_{j}}{\partial x^{3}}+\sum_{j=0}^{\infty} \frac{\partial^{3} v_{j}}{\partial y^{3}}\right]\right]
\end{gathered}
$$

for $j=0,1,2, .$.

$$
\begin{aligned}
& v_{1}(x, y, t)=-\mathcal{L}^{-1}\left[\frac{1}{s^{\gamma}} \mathcal{L}\left[\frac{\partial^{3} v_{0}}{\partial x^{3}}+\frac{\partial^{3} v_{0}}{\partial y^{3}}\right]\right] \\
& v_{1}(x, y, t)=-2 \sin (x+y) \mathcal{L}^{-1}\left[\frac{1}{s^{\gamma+1}}\right]=-2 \sin (x+y) \frac{t^{\gamma}}{\Gamma(\gamma+1)} \\
& v_{2}(x, y, t)=-\mathcal{L}^{-1}\left[\frac{1}{s^{\gamma}} \mathcal{L}\left[\frac{\partial^{3} v_{1}}{\partial x^{3}}+\frac{\partial^{3} v_{1}}{\partial y^{3}}\right]\right] \\
& v_{2}(x, y, t)=-4 \cos (x+y) \mathcal{L}^{-1}\left[\frac{1}{s^{2 \gamma+1}}\right]=-4 \cos (x+y) \frac{t^{2 \gamma}}{\Gamma(2 \gamma+1)}
\end{aligned}
$$



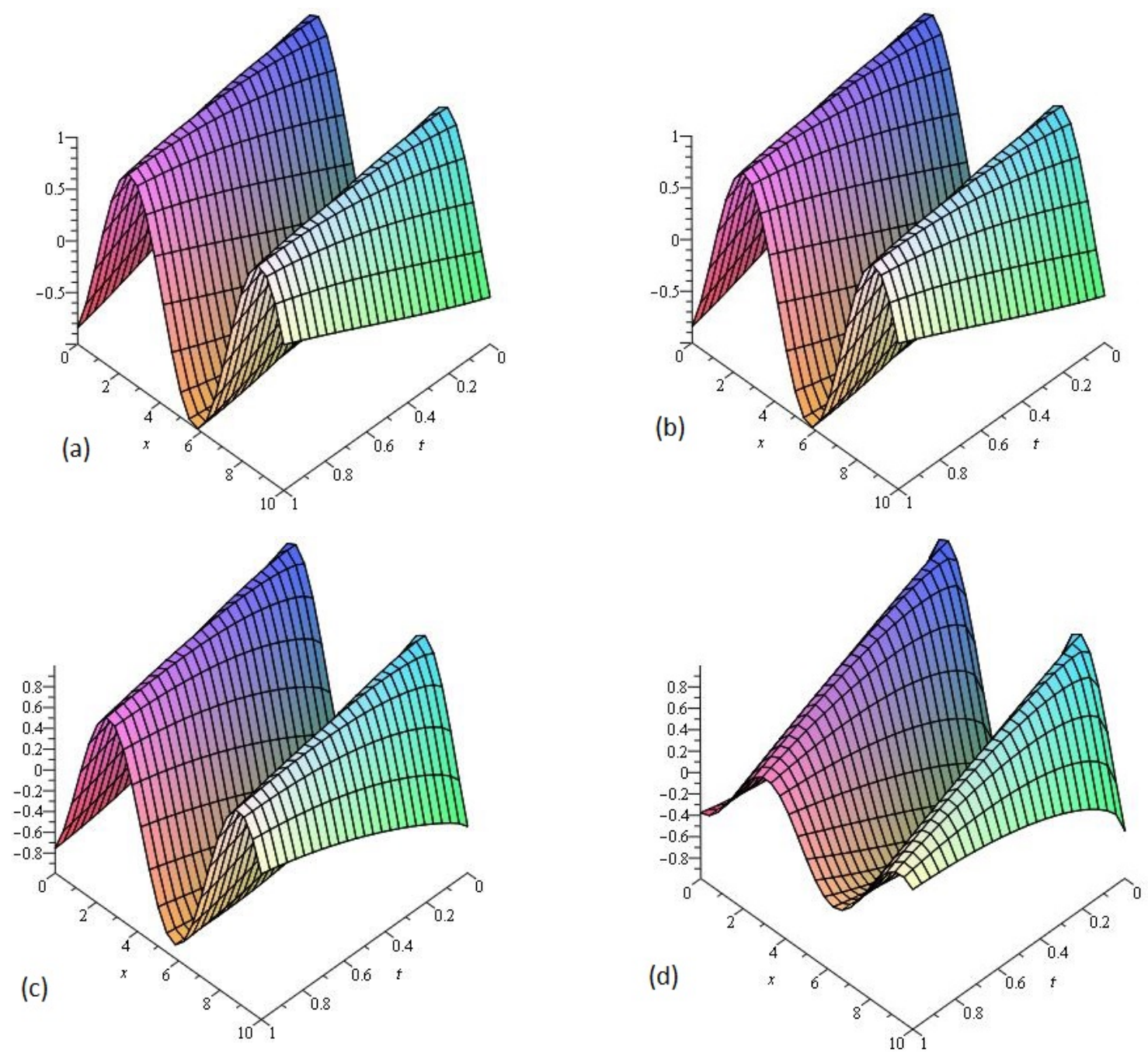

Figure 1. The (a) exact and (b) Laplace-Adomian decomposition method (LADM) solutions of $v(x, t)$ of Example 1, at $\gamma=1$. The LADM solution of $v(x, t)$ of Example 1 , at (c) $\gamma=0.75$ and (d) $\gamma=0.50$.

The subsequent terms are

$$
\begin{aligned}
& v_{3}(x, y, t)=-\mathcal{L}^{-1}\left[\frac{1}{s^{\gamma}} \mathcal{L}\left[\frac{\partial^{3} v_{2}}{\partial^{3} x}+\frac{\partial^{3} v_{2}}{\partial y^{3}}\right]\right] \\
& v_{3}(x, y, t)=8 \sin (x+y) \mathcal{L}^{-1}\left[\frac{1}{s^{3 \gamma+1}}\right]=8 \sin (x+y) \frac{t^{3 \gamma}}{\Gamma(3 \gamma+1)} .
\end{aligned}
$$

The LADM solution for Example 2 is

$$
\begin{gathered}
v(x, y, t)=v_{0}(x, y, t)+v_{1}(x, y, t)+v_{2}(x, y, t)+v_{3}(x, y, t)+\ldots, \\
v(x, y, t)=\cos (x+y)-2 \sin (x+y) \frac{t^{\gamma}}{\Gamma(\gamma+1)}-4 \cos (x+y) \frac{t^{2 \gamma}}{\Gamma(2 \gamma+1)} \\
+8 \sin (x+y) \frac{t^{3 \gamma}}{\Gamma(3 \gamma+1)}+\ldots
\end{gathered}
$$


and so on. The solution in a series form is given by

$$
\begin{aligned}
& v(x, y, t)=\cos (x+y)\left(1-\frac{4 t^{2 \gamma}}{\Gamma(2 \gamma+1)}+\frac{16 t^{2 \gamma}}{\Gamma(2 \gamma+1)}-\ldots\right) \\
& -\sin (x+y)\left(\frac{2 t^{\gamma}}{\Gamma(\gamma+1)}-\frac{8 t^{3 \gamma}}{\Gamma(3 \gamma+1)}+\frac{32 t^{5 \gamma}}{\Gamma(5 \gamma+1)}-\ldots\right),
\end{aligned}
$$

when $\gamma=1$, then LADM solution is

$$
v(x, y, t)=\cos (x+y+2 t) .
$$

Figure 2 consists of four graphs; (a) the exact solution of $v(x, y, t)$ and (b) LADM solution $v(x, y, t)$ of Example 2 at $\gamma=1$. Figure $2 \mathrm{a}$, b indicate that the present method has strong agreement with exact solution for the problem. In Figure 2c,d, two graphs (c) and (d) are given, that represents the analytical solution of Example 2 at fractional $\gamma=0.75$ and 0.50 respectively. Figure $2 c, d$ reflects that as fractional order approaches to integer order the solution surfaces of fractional order are convergent to the integer order surface. It means that physically we can model any of the surfaces as desired by the physical phenomena occurring in nature.

Example 3. Consider the following non-homogeneous fractional dispersive KdV equation [33]

$$
\frac{\partial^{\gamma} v}{\partial t^{\gamma}}+\frac{\partial^{3} v}{\partial x^{3}}=-\sin \pi x \sin t-\pi^{3} \cos \pi x \cos t, \quad 0<\gamma \leq 1,
$$

with initial condition

$$
v(x, 0)=\sin \pi x
$$

Taking Laplace transform of Equation (32),

$$
\begin{gathered}
\mathcal{L}\left[\frac{\partial^{\gamma} v}{\partial t^{\gamma}}\right]=\mathcal{L}\left[-\sin \pi x \sin t-\pi^{3} \cos \pi x \cos t\right]-\mathcal{L}\left[\frac{\partial^{3} v}{\partial x^{3}}\right], \\
s^{\gamma} \mathcal{L}[v(x, t)]-s^{\gamma-1}[v(x, 0)]=\mathcal{L}\left[-\sin \pi x \sin t-\pi^{3} \cos \pi x \cos t\right]-\mathcal{L}\left[\frac{\partial^{3} v}{\partial x^{3}}\right] .
\end{gathered}
$$

Applying an inverse Laplace transform

$$
\begin{gathered}
v(x, t)=\mathcal{L}^{-1}\left[\frac{v(x, 0)}{s}+\frac{1}{s^{\gamma}} \mathcal{L}\left[-\sin \pi x \sin t-\pi^{3} \cos \pi x \cos t\right]-\frac{1}{s^{\gamma}} \mathcal{L}\left[\frac{\partial^{3} v}{\partial x^{3}}\right]\right], \\
v(x, t)=\mathcal{L}^{-1}\left[\frac{\sin \pi x}{s}\right]+\mathcal{L}^{-1}\left[\frac{1}{s^{\gamma}} \mathcal{L}\left[-\sin \pi x \sin t-\pi^{3} \cos \pi x \cos t\right]\right] \\
-\mathcal{L}^{-1}\left[\frac{1}{s^{\gamma}} \mathcal{L}\left[\frac{\partial^{3} v}{\partial x^{3}}\right]\right] .
\end{gathered}
$$



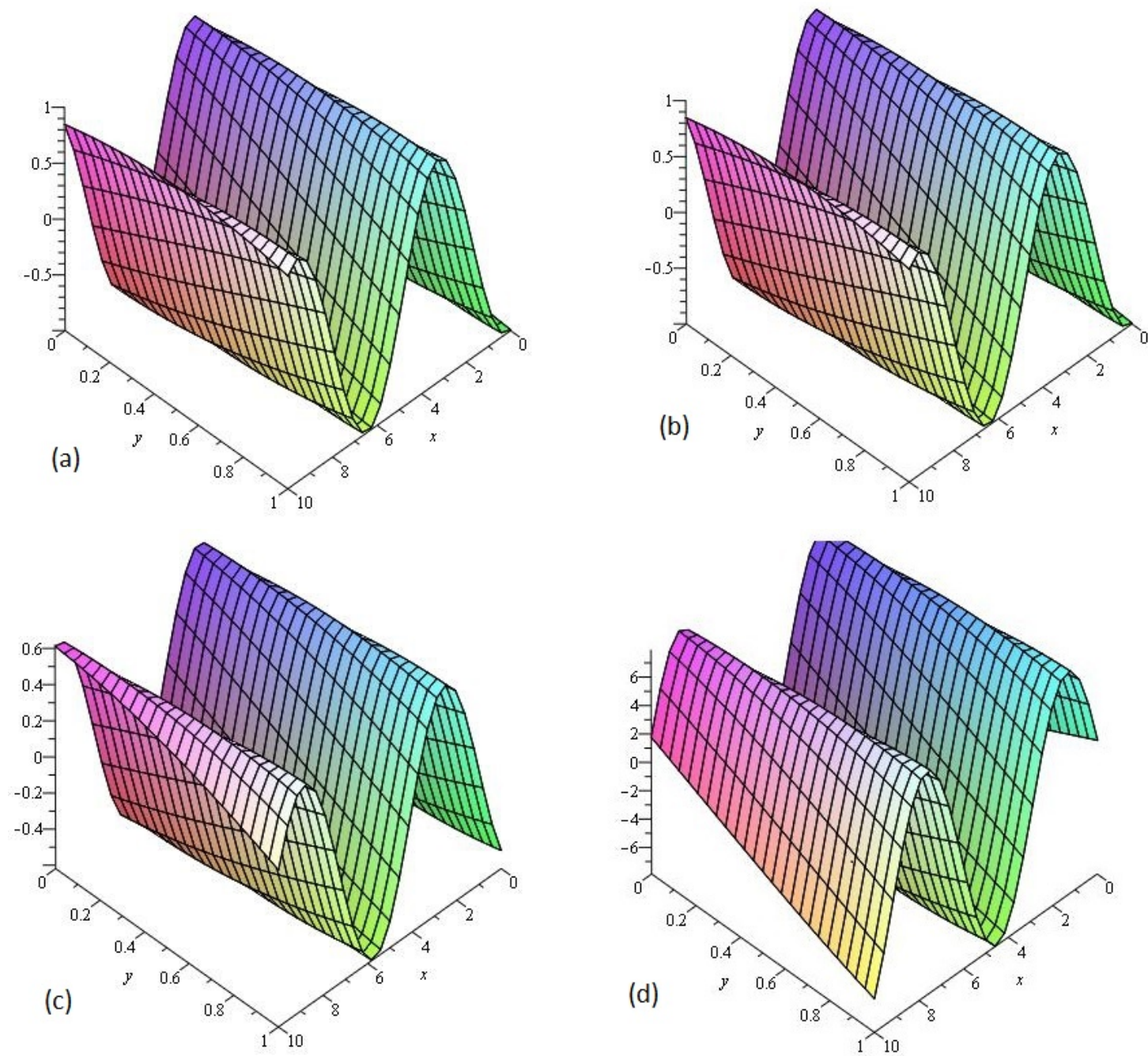

Figure 2. The (a) Exact and (b) LADM solutions of $v(x, y, t)$ of Example 2, at $\gamma=1$. The LADM solution of $v(x, y, t)$ of Example 2, at (c) $\gamma=0.75$ and (d) $\gamma=0.50$.

Using the ADM procedure, we get

$$
\begin{aligned}
& \sum_{j=0}^{\infty} v_{j}(x, t)=\mathcal{L}^{-1}\left[\frac{\sin \pi x}{s}\right]+\mathcal{L}^{-1}\left[\frac{1}{s^{\gamma}} \mathcal{L}\left[-\sin \pi x\left(t-\frac{t^{3}}{3 !}+\frac{t^{5}}{5 !}-\frac{t^{7}}{7 !}+\frac{t^{9}}{9 !}\right)\right]\right] \\
& +\mathcal{L}^{-1}\left[\frac{1}{s^{\gamma}} \mathcal{L}\left[-\pi^{3} \cos \pi x\left(1-\frac{t^{2}}{2 !}+\frac{t^{4}}{4 !}-\frac{t^{6}}{6 !}+\frac{t^{8}}{8 !}\right)\right]\right]-\mathcal{L}^{-1}\left[\frac{1}{s^{\gamma}} \mathcal{L}\left[\sum_{j=0}^{\infty} \frac{\partial^{3} v_{j}}{\partial x^{3}}\right]\right], \\
& v_{0}(x, t)=\sin \pi x \\
& -\sin \pi x\left(\frac{t^{\gamma+1}}{\Gamma(\gamma+2)}-\frac{t^{\gamma+3}}{\Gamma(\gamma+4)}+\frac{t^{\gamma+5}}{\Gamma(\gamma+6)}-\frac{t^{\gamma+7}}{\Gamma(\gamma+8)}+\frac{t^{\gamma+9}}{\Gamma(\gamma+10)}\right) \\
& -\pi^{3} \cos \pi x\left(\frac{t^{\gamma}}{\Gamma(\gamma+1)}-\frac{t^{\gamma+2}}{\Gamma(\gamma+3)}+\frac{t^{\gamma+4}}{\Gamma(\gamma+5)}-\frac{t^{\gamma+6}}{\Gamma(\gamma+7)}+\frac{t^{\gamma+8}}{\Gamma(\gamma+9)}\right), \\
& v_{j+1}(x, t)=-\mathcal{L}^{-1}\left[\frac{1}{s^{\gamma}} \mathcal{L}\left[\sum_{j=0}^{\infty} \frac{\partial^{3} v_{j}}{\partial x^{3}}\right]\right]
\end{aligned}
$$


for $j=0,1,2, .$.

$$
\begin{aligned}
& v_{1}(x, t)=-\mathcal{L}^{-1}\left[\frac{1}{s^{\gamma}} \mathcal{L}\left[\frac{\partial^{3} v_{0}}{\partial x^{3}}\right]\right], \\
& v_{1}(x, t)=\pi^{3} \cos \pi x \frac{t^{\gamma}}{\Gamma(\gamma+1)} \\
& -\pi^{3} \cos \pi x\left(\frac{t^{2 \gamma+1}}{\Gamma(2 \gamma+2)}-\frac{t^{2 \gamma+3}}{\Gamma(2 \gamma+4)}+\frac{t^{2 \gamma+5}}{\Gamma(2 \gamma+6)}-\frac{t^{2 \gamma+7}}{\Gamma(2 \gamma+8)}+\frac{t^{2 \gamma+9}}{\Gamma(2 \gamma+10)}\right) \\
& +\pi^{6} \sin \pi x\left(\frac{t^{2 \gamma}}{\Gamma(2 \gamma+1)}-\frac{t^{2 \gamma+2}}{\Gamma(2 \gamma+3)}+\frac{t^{2 \gamma+4}}{\Gamma(2 \gamma+5)}-\frac{t^{2 \gamma+6}}{\Gamma(2 \gamma+7)}+\frac{t^{2 \gamma+8}}{\Gamma(2 \gamma+9)}\right) \\
& v_{2}(x, t)=-\mathcal{L}^{-1}\left[\frac{1}{s^{\gamma}} \mathcal{L}\left[\frac{\partial^{3} v_{1}}{\partial x^{3}}\right]\right], \\
& v_{2}(x, t)=-\pi^{6} \sin \pi x \frac{t^{2} \gamma}{\Gamma(2 \gamma+1)} \\
& +\pi^{6} \sin \pi x\left(\frac{t^{3 \gamma+1}}{\Gamma(3 \gamma+2)}-\frac{t^{3 \gamma+3}}{\Gamma(3 \gamma+4)}+\frac{t^{3 \gamma+5}}{\Gamma(3 \gamma+6)}-\frac{t^{3 \gamma+7}}{\Gamma(3 \gamma+8)}+\frac{t^{3 \gamma+9}}{\Gamma(3 \gamma+10)}\right) \\
& +\pi^{9} \cos \pi x\left(\frac{t^{3 \gamma}}{\Gamma(3 \gamma+1)}-\frac{t^{3 \gamma+2}}{\Gamma(3 \gamma+3)}+\frac{t^{3 \gamma+4}}{\Gamma(3 \gamma+5)}-\frac{t^{3 \gamma+6}}{\Gamma(3 \gamma+7)}+\frac{t^{3 \gamma+8}}{\Gamma(3 \gamma+9)}\right) .
\end{aligned}
$$

The LADM solution for Example 3 is

$$
\begin{gathered}
v(x, t)=v_{0}(x, t)+v_{1}(x, t)+v_{2}(x, t)+v_{3}(x, t)+\ldots \\
v(x, t)=\sin \pi x \\
-\sin \pi x\left(\frac{t^{\gamma+1}}{\Gamma(\gamma+2)}-\frac{t^{\gamma+3}}{\Gamma(\gamma+4)}+\frac{t^{\gamma+5}}{\Gamma(\gamma+6)}-\frac{t^{\gamma+7}}{\Gamma(\gamma+8)}+\frac{t^{\gamma+9}}{\Gamma(\gamma+10)}\right) \\
-\pi^{3} \cos \pi x\left(\frac{t^{\gamma}}{\Gamma(\gamma+1)}-\frac{t^{\gamma+2}}{\Gamma(\gamma+3)}+\frac{t^{\gamma+4}}{\Gamma(\gamma+5)}-\frac{t^{\gamma+6}}{\Gamma(\gamma+7)}+\frac{t^{\gamma+8}}{\Gamma(\gamma+9)}\right) \\
+\pi^{3} \cos \pi x \frac{t^{\gamma}}{\Gamma(\gamma+1)} \\
-\pi^{3} \cos \pi x\left(\frac{t^{2 \gamma+1}}{\Gamma(2 \gamma+2)}-\frac{t^{2 \gamma+3}}{\Gamma(2 \gamma+4)}+\frac{t^{2 \gamma+5}}{\Gamma(2 \gamma+6)}-\frac{t^{2 \gamma+7}}{\Gamma(2 \gamma+8)}+\frac{t^{2 \gamma+9}}{\Gamma(2 \gamma+10)}\right) \\
+\pi^{6} \sin \pi x\left(\frac{t^{2 \gamma}}{\Gamma(2 \gamma+1)}-\frac{t^{2 \gamma+2}}{\Gamma(2 \gamma+3)}+\frac{t^{2 \gamma+4}}{\Gamma(2 \gamma+5)}-\frac{t^{2 \gamma+6}}{\Gamma(2 \gamma+7)}+\frac{t^{2 \gamma+8}}{\Gamma(2 \gamma+9)}\right) \\
-\pi^{6} \sin \pi x \frac{t^{2} \gamma}{\Gamma(2 \gamma+1)} \\
+\pi^{6} \sin \pi x\left(\frac{t^{3 \gamma+1}}{\Gamma(3 \gamma+2)}-\frac{t^{3 \gamma+3}}{\Gamma(3 \gamma+4)}+\frac{t^{3 \gamma+5}}{\Gamma(3 \gamma+6)}-\frac{t^{3 \gamma+7}}{\Gamma(3 \gamma+8)}+\frac{t^{3 \gamma+9}}{\Gamma(3 \gamma+10)}\right) \\
+\pi^{9} \cos \pi x\left(\frac{t^{3 \gamma}}{\Gamma(3 \gamma+1)}-\frac{t^{3 \gamma+2}}{\Gamma(3 \gamma+3)}+\frac{t^{3 \gamma+4}}{\Gamma(3 \gamma+5)}-\frac{t^{3 \gamma+6}}{\Gamma(3 \gamma+7)}+\frac{t^{3 \gamma+8}}{\Gamma(3 \gamma+9)}\right)+\ldots
\end{gathered}
$$

when $\gamma=1$, then the LADM solution is

$$
v(x, t)=\sin \pi x \cos t .
$$

Figure 3 consists of three graphs; (a) the exact solution of $v(x, t)$ and (b) LADM solution $v(x, t)$ of Example 3 at $\gamma=1$. Figure $3 a, b$ indicate that the present method has strong agreement with exact solution for the problem. In Figure 3c, graphs (c) are given, that represent the analytical solution of Example 3 at fractional $\gamma=0.75$ respectively. Figure $3 \mathrm{c}$ reflects that the fractional order approaches to integer order, the solution surfaces of fractional order are convergent to the integer order surface. 
It means that physically we can model, any of the surfaces as desired by the physical phenomena occurring in nature.
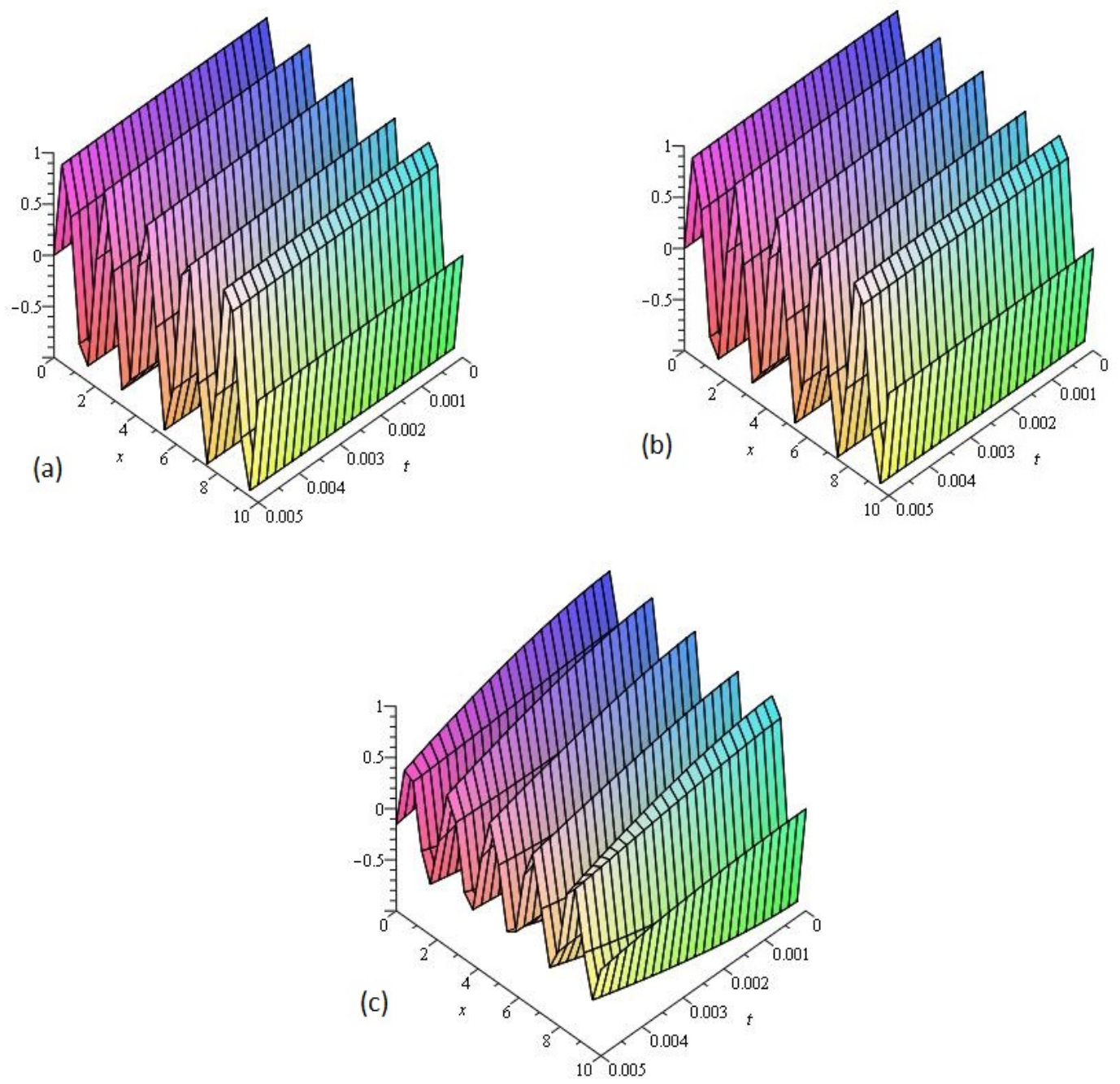

Figure 3. The (a) Exact and (b) LADM solutions of $v(x, t)$ of Example 3, at $\gamma=1$. The LADM solution of $v(x, t)$ of Example 3, at (c) $\gamma=0.75$.

Example 4. Consider the following non-homogeneous fractional dispersive KdV equation in three dimensional space [33]

$$
\begin{aligned}
& \frac{\partial^{\gamma} v}{\partial t^{\gamma}}+\frac{\partial^{3} v}{\partial x^{3}}+\frac{1}{8} \frac{\partial^{3} v}{\partial y^{3}}+\frac{1}{27} \frac{\partial^{3} v}{\partial z^{3}}=-\sin (x+2 y+3 z) \cos t \\
& +\sin (x+2 y+3 z) \cos t, \quad t>0, \quad 0<\gamma \leq 1
\end{aligned}
$$

with the initial condition

$$
v(x, y, z, 0)=0
$$

Taking Laplace transform of Equation (37),

$$
\begin{aligned}
& \mathcal{L}\left[\frac{\partial^{\gamma} v}{\partial t^{\gamma}}\right]=\mathcal{L}[\sin (x+2 y+3 z) \cos t]-\mathcal{L}[3 \cos (x+2 y+3 z) \sin t] \\
& -\mathcal{L}\left[\frac{\partial^{3} v}{\partial x^{3}}+\frac{1}{8} \frac{\partial^{3} v}{\partial y^{3}}+\frac{1}{27} \frac{\partial^{3} v}{\partial z^{3}}\right],
\end{aligned}
$$




$$
\begin{aligned}
& s^{\gamma} \mathcal{L}[v(x, y, z, t)]-s^{\gamma-1}[v(x, y, z, 0)]=\mathcal{L}[\sin (x+2 y+3 z) \cos t] \\
& -\mathcal{L}[3 \cos (x+2 y+3 z) \sin t]-\mathcal{L}\left[\frac{\partial^{3} v}{\partial x^{3}}+\frac{1}{8} \frac{\partial^{3} v}{\partial y^{3}}+\frac{1}{27} \frac{\partial^{3} v}{\partial z^{3}}\right] .
\end{aligned}
$$

Applying inverse Laplace transform

$$
\begin{aligned}
& v(x, y, z, t)=\mathcal{L}^{-1}\left[\frac{v(x, y, z, 0)}{s}+\frac{1}{s^{\gamma}} \mathcal{L}[\sin (x+2 y+3 z) \cos t]\right] \\
& +\mathcal{L}^{-1}\left[\frac{1}{s^{\gamma}} \mathcal{L}[-3 \cos (x+2 y+3 z) \sin t]\right]-\mathcal{L}^{-1}\left[\frac{1}{s^{\gamma}} \mathcal{L}\left[\frac{\partial^{3} v}{\partial x^{3}}+\frac{1}{8} \frac{\partial^{3} v}{\partial y^{3}}+\frac{1}{27} \frac{\partial^{3} v}{\partial z^{3}}\right]\right], \\
& v_{0}(x, y, z, t)=\mathcal{L}^{-1}\left[\frac{1}{s^{\gamma}} \mathcal{L}\left[\sin (x+2 y+3 z)\left(1-\frac{t^{2}}{2 !}+\frac{t^{4}}{4 !}-\frac{t^{6}}{6 !}+\frac{t^{8}}{8 !}\right)\right]\right] .
\end{aligned}
$$

Using the ADM procedure, we get

$$
\begin{aligned}
& \sum_{j=0}^{\infty} v_{j}(x, y, t)=\mathcal{L}^{-1}\left[\frac{1}{s^{\gamma}} \mathcal{L}\left[-3 \cos (x+2 y+3 z)\left(t-\frac{t^{3}}{3 !}+\frac{t^{5}}{5 !}-\frac{t^{7}}{7 !}+\frac{t^{9}}{9 !}\right)\right]\right] \\
& -\mathcal{L}^{-1}\left[\frac{1}{s^{\gamma}} \mathcal{L}\left[\sum_{j=0}^{\infty} \frac{\partial^{3} v_{j}}{\partial x^{3}}+\frac{1}{8} \sum_{j=0}^{\infty} \frac{\partial^{3} v_{j}}{\partial y^{3}}+\frac{1}{27} \sum_{j=0}^{\infty} \frac{\partial^{3} v_{j}}{\partial z^{3}}\right]\right], \\
& v_{0}(x, y, z, t)= \\
& \sin (x+2 y+3 z)\left(\frac{t^{\gamma}}{\Gamma(\gamma+1)}-\frac{t^{\gamma+2}}{\Gamma(\gamma+3)}+\frac{t^{\gamma+4}}{\Gamma(\gamma+5)}-\frac{t^{\gamma+6}}{\Gamma(\gamma+7)}+\frac{t^{\gamma+8}}{\Gamma(\gamma+9)}\right), \\
& v_{1}(x, y, z, t)=\mathcal{L}^{-1}\left[\frac{1}{s^{\gamma}} \mathcal{L}\left[-3 \cos (x+2 y+3 z)\left(t-\frac{t^{3}}{3 !}+\frac{t^{5}}{5 !}-\frac{t^{7}}{7 !}+\frac{t^{9}}{9 !}\right)\right]\right] \\
& -\mathcal{L}^{-1}\left[\frac{1}{s^{\gamma}} \mathcal{L}\left[\frac{\partial^{3} v_{0}}{\partial x^{3}}+\frac{1}{8} \frac{\partial^{3} v_{0}}{\partial y^{3}}+\frac{1}{27} \frac{\partial^{3} v_{0}}{\partial z^{3}}\right]\right], \\
& v_{j+1}(x, y, z, t)=-\mathcal{L}^{-1}\left[\frac{1}{s^{\gamma}} \mathcal{L}\left[\sum_{j=0}^{\infty} \frac{\partial^{3} v_{j}}{\partial x^{3}}+\frac{1}{8} \sum_{j=0}^{\infty} \frac{\partial^{3} v_{j}}{\partial y^{3}}+\frac{1}{27} \sum_{j=0}^{\infty} \frac{\partial^{3} v_{j}}{\partial z^{3}}\right]\right],
\end{aligned}
$$

for $j=0,1,2, \ldots$

$$
\begin{aligned}
& v_{1}(x, y, z, t)=0, \\
& v_{j+1}(x, y, z, t)=0 .
\end{aligned}
$$

This readily yields the exact solution

$$
\begin{aligned}
& v(x, y, z, t)= \\
& \sin (x+2 y+3 z)\left(\frac{t^{\gamma}}{\Gamma(\gamma+1)}-\frac{t^{\gamma+2}}{\Gamma(\gamma+3)}+\frac{t^{\gamma+4}}{\Gamma(\gamma+5)}-\frac{t^{\gamma+6}}{\Gamma(\gamma+7)}+\frac{t^{\gamma+8}}{\Gamma(\gamma+9)}\right)
\end{aligned}
$$

when $\gamma=1$, then LADM solution is

$$
v(\mathrm{x}, \mathrm{y}, \mathrm{t})=\sin (\mathrm{x}+2 \mathrm{y}+3 \mathrm{z}) \sin \mathrm{t} .
$$

Figure 4 consists of two graphs; (a) the exact solution of $v(x, y, t)$ and (b) the LADM solution $v(x, y, t)$ of Example 4 at $\gamma=1$. Figure $4 \mathrm{a}, \mathrm{b}$ indicate that the present method has strong agreement with exact solution for the problem. In Figure 4c,d, two graphs (c) and (d) are given, that represent the analytical solution of Example 4 at fractional $\gamma=0.75$ and 0.50 respectively. Figure $4 c, d$ reflects that, as fractional order approaches to integer order, the solution surfaces of fractional order are convergent 
to the integer order surface. It means that physically we can model, any of the surfaces as desired by the physical phenomena occurring in nature.
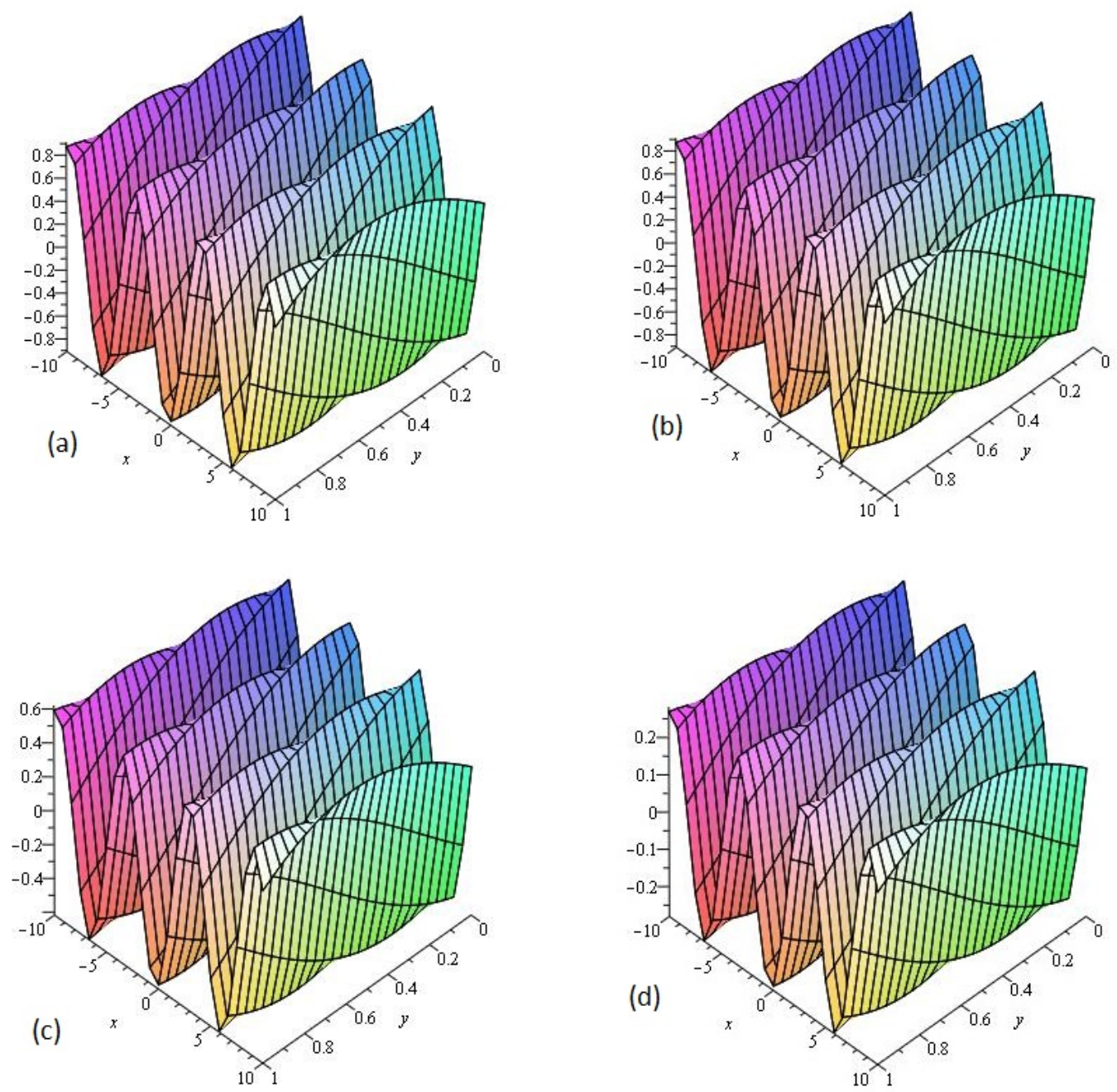

Figure 4. The (a) exact and (b) LADM solutions of $v(x, y, z, t)$ of Example 4 , at $\gamma=1$. The LADM solution of $v(x, y, z, t)$ of Example 4 , at (c) $\gamma=0.75$ and (d) $\gamma=0.50$.

\section{Conclusions}

In this paper, the analytical solutions of third order dispersive fractional partial differential equations are determined, using LADM. The fractional derivatives are described by the Caputo operator. The LADM, solutions are obtained at fractional and integer orders for all problems. The results revealed the highest agreement with the exact solutions for the problems. The LADM solutions for some numerical examples have shown the validity of the proposed method. It is also investigated that the fractional order solutions are convergent to the exact solution for the problems as fractional order approaches to integer order. The implementation of LADM to illustrative examples have also confirmed that the fractional order mathematical model can be the best representation of any experimental data as compare to integer order model. Moreover, by taking different fractional orders, we can find a way to set suitable mathematical model for any experimental data, and thus found reasonable consequences. Hence, it is concluded that LADM is the best tool for the solution of FPDEs, as compare to ADM, VIM and DTM in literature. LADM provide the highest rate of convergence to the exact solution for the problems. In future, LADM can be used to find the analytical solution of other non-linear FPDEs, which are frequently used in science and engineering. LADM, solutions for 
fractional order problems will prove the better understanding of the real world problems represented by FPDEs.

Author Contributions: Conceptualization, R.S. and H.K.; Methodology, M.A.; Software, R.S.; Validation, H.K. and M.A.; Formal Analysis, R.S.; Investigation, M.A. and P.K.; Resources, H.K. and P.K.; Data Curation, R.S.; Writing_-Original Draft Preparation, R.S.; Writing—Review and Editing, H.K., M.A. and P.K.; Visualization, M.A.; Supervision, M.A., P.K.; Project Administration, P.K.; Funding Acquisition, P.K.

Funding: Center of Excellence in Theoretical and Computational Science (TaCS-CoE) Faculty of Science, King Mongkuts University of Technology Thonburi (KMUTT).

Conflicts of Interest: The authors have no conflict of interest.

\section{Abbreviations}

The following abbreviations are used in this manuscript:

$\begin{array}{ll}\text { LADM } & \text { Laplace-Adomian decomposition method } \\ \text { PDE } & \text { Partial differential equation } \\ \text { KdV } & \text { Korteweg-de Vries } \\ \text { LHAM } & \text { Laplace homotopy analysis method } \\ \text { FVIM } & \text { Fractional variational iteration method } \\ \text { FDTM } & \text { Fractional differential transform method } \\ \text { HASTM } & \text { Homotopy analysis Sumudu transform method } \\ \text { DE } & \text { Differential equation } \\ \text { SM } & \text { Spline method }\end{array}$

\section{References}

1. Goswami, A.; Singh, J.; Kumar, D. Numerical simulation of fifth order Kdv equations occurring in magneto-acoustic waves. Ain Shams Eng. J. 2018, 9, 2265-2273. [CrossRef]

2. Steudel, H.; Drazin, P.G.; Johnson, R.S. Solitons: An Introduction. Cambridge etc., Cambridge University Press 1989. XII, 226 pp., $£ 11.95$ P/b. ISBN 0-521-33655-4 (Cambridge Texts in Applied Mathematics). ZAMM-J. Appl. Math. Mech./Zeitschrift für Angewandte Mathematik und Mechanik 1990, 70, 340. [CrossRef]

3. Djidjeli, K.; Price, W.G.; Twizell, E.H.; Wang, Y. Numerical methods for the solution of the third-and fifth-order dispersive Korteweg-de Vries equations. J. Comput. Appl. Math. 1995, 58, 307-336. [CrossRef]

4. Zahran, M.A.; El-Shewy, E.K. Contribution of Higher-Order Dispersion to Nonlinear Electron-Acoustic Solitary Waves in a Relativistic Electron Beam Plasma System. Phys. Scr. 2008, 78. [CrossRef]

5. Seadawy, A.R. New exact solutions for the KdV equation with higher order nonlinearity by using the variational method. Comput. Math. Appl. 2011, 62, 3741-3755. [CrossRef]

6. Shi, Y.; Xu, B.; Guo, Y. Numerical solution of Korteweg-de Vries-Burgers equation by the compact-type CIP method. Adv. Differ. Equ. 2015, 2015, 353. [CrossRef]

7. Ubriaco, M.R. Entropies based on fractional calculus. Phys. Lett. A 2009, 373, 2516-2519. [CrossRef]

8. Machado, J.T. Entropy analysis of integer and fractional dynamical systems. Nonlinear Dyn. 2010, 62, 371-378.

9. Ball, J.M.; Chen, G.Q.G. Entropy and convexity for nonlinear partial differential equations. Philos. Trans. R. Soc. A Math. Phys. Eng. Sci. 2013. [CrossRef]

10. Sibatov, R.; Shulezhko, V.; Svetukhin, V. Fractional Derivative Phenomenology of Percolative Phonon-Assisted Hopping in Two-Dimensional Disordered Systems. Entropy 2017, 19, 463. [CrossRef]

11. Lopes, A.M.; Tenreiro Machado, J.A. Entropy Analysis of Soccer Dynamics. Entropy 2019, 21, 187. [CrossRef]

12. Jiang, J.; Feng, Y.; Li, S. Exact Solutions to the Fractional Differential Equations with Mixed Partial Derivatives. Axioms 2018, 7, 10. [CrossRef]

13. Silva, F.; Moreira, D.; Moret, M. Conformable Laplace Transform of Fractional Differential Equations. Axioms 2018, 7, 55. [CrossRef]

14. Yavuz, M.; Özdemir, N. European vanilla option pricing model of fractional order without singular kernel. Fractal Fract. 2018, 2, 3. [CrossRef]

15. Thabet, H.; Kendre, S.; Chalishajar, D. New analytical technique for solving a system of nonlinear fractional partial differential equations. Mathematics 2017, 5, 47. [CrossRef] 
16. Yépez-Martínez, H.; Gómez-Aguilar, F.; Sosa, I.O.; Reyes, J.M.; Torres-Jiménez, J. The Feng's first integral method applied to the nonlinear $\mathrm{mKdV}$ space-time fractional partial differential equation. Revista Mexicana de FíSica 2016, 62, 310-316.

17. Prakash, A.; Kumar, M. Numerical method for fractional dispersive partial differential equations. Commun. Numer. Anal. 2017, 1, 1-18. [CrossRef]

18. Kocak, H.; Pinar, Z. On solutions of the fifth-order dispersive equations with porous medium type non-linearity. Waves Random Complex Media 2018, 28, 516-522. [CrossRef]

19. Kanth, A.R. and Aruna, K. Solution of fractional third-order dispersive partial differential equations. Egypt. J. Basic Appl. Sci. 2015, 2, 190-199. [CrossRef]

20. Sultana, T.; Khan, A.; Khandelwal, P. A new non-polynomial spline method for solution of linear and non-linear third order dispersive equations. Adv. Differ. Equ. 2018, 2018, 316. [CrossRef]

21. Pandey, R.K.; Mishra, H.K. Homotopy analysis Sumudu transform method for time-Fractional third order dispersive partial differential equation. Adv. Comput. Math. 2017, 43, 365-383. [CrossRef]

22. Jafari, H.; Khalique, C.M.; Nazari, M. Application of the Laplace decomposition method for solving linear and nonlinear fractional diffusion-wave equations. Appl. Math. Lett. 2011, 24, 1799-1805. [CrossRef]

23. Mohamed, M.Z. Comparison between the Laplace Decomposition Method and Adomian Decomposition in Time-Space Fractional Nonlinear Fractional Differential Equations. Appl. Math. 2018, 9, 448. [CrossRef]

24. Gaxiola, O.G. The Laplace-Adomian decomposition method applied to the Kundu-Eckhaus equation. Int. J. Math. Its Appl. 2017, 5, 1-12.

25. Al-Zurigat, M. Solving nonlinear fractional differential equation using a multi-step Laplace Adomian decomposition method. Ann. Univ. Craiova-Math. Comput. Sci. Ser. 2012, 39, 200-210.

26. Haq, F.; Shah, K.; ur Rahman, G.; Shahzad, M. Numerical solution of fractional order smoking model via laplace Adomian decomposition method. Alex. Eng. J. 2018, 57, 1061-1069. [CrossRef]

27. Miller, K.S.; Ross, B. An Introduction to the Fractional Calculus and Fractional Differential Equations; Wiley: New York, NY, USA, 1993.

28. Hilfer, R. Applications of Fractional Calculus in Physics; World Science Publishing: River Edge, NJ, USA, 2000.

29. Podlubny, I. Fractional Differential Equations: An Introduction to Fractional Derivatives, Fractional Differential Equations, to Methods of Their Solution and Some of Their Applications; Elsevier: Academic Press: San Diego, CA, USA, 1998; Volume 198.

30. Gómez-Aguilar, J.F.; Torres, L.; Yépez-Martínez, H.; Baleanu, D.; Reyes, J.M.; Sosa, I.O. Fractional Liénard type model of a pipeline within the fractional derivative without singular kernel. Adv. Differ. Equ. 2016, 2016, 173. [CrossRef]

31. Morales-Delgado, V.F.; Taneco-Hernández, M.A.; Gómez-Aguilar, J.F. On the solutions of fractional order of evolution equations. Eur. Phys. J. Plus 2017, 132, 47. [CrossRef]

32. Naghipour, A.; Manafian, J. Application of the Laplace Adomian decomposition and implicit methods for solving Burgers' equation. TWMS J. Pure Appl. Math. 2015, 6, 68-77.

33. Wazwaz, A.M. An analytic study on the third-order dispersive partial differential equations. Appl. Math. Comput. 2003, 142, 511-520. [CrossRef]

(c) 2019 by the authors. Licensee MDPI, Basel, Switzerland. This article is an open access article distributed under the terms and conditions of the Creative Commons Attribution (CC BY) license (http://creativecommons.org/licenses/by/4.0/). 